\title{
Halitosis: the role of the gastroenterologist
}

\begin{abstract}
For years there is controversy about the role played by the gastroenterologist in the diagnosis and treatment of halitosis. Vague indications upper gastrointestinal endoscopy, as well as the inadequate indication of tomographic and functional studies of the upper gastrointestinal tract have determined the need to establish a good practice in terms of Halitosis and the real need that exists that a patient be evaluated by the gastroenterologist, as well as other medical specialties.
\end{abstract}

Keywords: Halitosis, Bad Breath, Oral Odor, Gastroenterologist, Gastrointestinal Endoscopy
Volume 5 Issue 6 - 2016

\section{Daniel Tepedino Peluso}

Department of Gastroenterology, Hospital Universitario de Caracas, Venezuela

Correspondence: Daniel Tepedino Peluso, C.I.VI5.646.405.Av. Humboldt cruce con Calle Baldo Urb. Bello Monte, Residencia Crystal Plaza, piso 4,Apto 4-B, Parroquia El Recreo, Municipio Libertador, Código postal 1050, Caracas - Venezuela ,Tel +58412707/462, Email d.tepedino@gmail.com

Received: July 28, 2016 | Published: December 0I, 2016

\section{Introduction}

Over time the number of patients who consult Gastroenterologist by Halitosis has been increasing. In the mind of Gastroenterologist during the consult are generated questions like: Halitosis is a disease that must be handled by my specialty an endoscopy is required to diagnose it? How can I help this patient ?, two or more specialists are needed for proper management?

Halitosis is an important disease, it is estimated that about 3\% of the world population has presented at some point in their life, it causes anxiety and embarrassment to the sufferer. Odor or bad breath is caused by alterations in the balance in the oral bacterial microflora, usually by uncontrolled growth of anaerobic bacteria which results in the production and exhalation of malodorous gases associated with the presence of volatile sulfur compounds, primarily hydrogen sulfide and methylmercaptan. ${ }^{1}$

Among the nonoral etiologies of bad breath, the nasal passages predominate. In such cases, the telltale odor can be smelled most strongly from the nose, rather than the mouth. Nasal odor may be indicative of nasal infection or a problem affecting airflow associated with thick mucous secretions. ${ }^{2-4}$

Halitosis was described by many cultures, it was associated with poor oral hygiene. Attempts to treat include chewing tubers (Latin America), eggshells (China) and leaves of plants such as mint and basil (Italy)..$^{5-7}$ Most research shows association between poor salivation, chronic infections in the oral cavity and a deep hairy surface on the back of the tongue as facilitators of Halitosis. One report even suggests that emotional factors also influence its development. ${ }^{8}$

Many nonoral systemic conditions, such as kidney failure, bronchial and lung infections, metabolic dysfunctions, various carcinomas, and biochemical disorders, can result in bad breath, but all these taken together account for only a very small percentage. Generally, the incidence of systemic disorders causing for oral malodor (bad breath) amounts to only $1-2 \%$ of the halitosis clinic visits.

Contrary to common thinking, bad breath (halitosis) from the gastrointestinal tract is considered to be extremely rare. The Esophagus is normally collapsed and closed, and whereas the occasional belch may carry some odor up from the stomach, the possibility of gastric air reflux continuously escaping from the mouth and nose is very remote. ${ }^{3,9-11}$
Although there are multiple scales to measure the intensity of halitosis, organoleptic characteristics and devices that can measure the volatile compounds that cause it and even quantify objectively, many specialists insist on using their own nose.

There are reports that assess the patient's perception about their Halitosis and several show a poor correlation between the intensity reported by the patient and perceived by the specialist. ${ }^{12}$ Despite providing adequate treatment, improve oral hygiene, use more and better toothpastes, mouthwashes, tongue brushes and dental floss, improvement of Halitosis could not be total.

High protein diets, frequent and abundant water consumption, use of medicated sprays and quitting smoking are part of comprehensive treatment of halitosis. ${ }^{13}$ The dentist should be responsible for providing primary care patient and rule out any cause of oral disease first.

Otolaryngologist involvement should be considered when symptoms, signs or findings suggest that nasal-pharyngeal disorders may be responsible for halitosis. In patients with symptoms such as dysphagia, regurgitation, epigastric pain, dyspepsia and heartburn should be recommended consulting the Gastroenterologist considering that the existence of such symptoms could not be the cause of halitosis, but rather, coexistence with other gastrointestinal diseases whose association with bad breath is still questionable. The gastroenterologist must decide the need for upper endoscopy, imaging or functional studies of the upper digestive tract according to the symptoms, signs, findings and considerations.

\section{Conclusion}

Primary evaluation by a gastroenterologist is not justified, it is not cost-effective and is a poor clinical practice that delay diagnosis and treatment of Halitosis. The probability that a metabolic disease or lower respiratory tract infection is the cause of Halitosis is very low, however, if the patient has any relevant personal or family medical history should suggest evaluation by internal medicine or a pulmonologist, in the absence of other symptoms or signs may be useful to exclude these diagnoses.

The patient should be duly informed of the possible causes of Halitosis and not just referred to another specialist detaching the primary handler, which is and will be the dentist while direct correlation or causality is proved with some non-oral pathology. In the light of that reported in the literature, in the absence of conclusive 
evidence to guide on a gastrointestinal cause, Gastroenterologist is not the person who initially should be consulted or who has the main role in the diagnosis and treatment of halitosis.

\section{Acknowledgements}

The author want to thank all the staff of Gastroenterology Department of the Hospital Universitario de Caracas.

\section{References}

1. Laleman I, Dadamio J, De Geest S, et al. Instrumental assessment of halitosis for the general dental practitioner. $J$ Breath Res. 2014;8(1):017103.

2. Preti G, Clark L, Cowart BJ, et al. Non-oral etiologies of oral malodor and altered chemosensation. J Periodontol. 1992;63(9):790-796.

3. Finkelstein Y. The otolaryngologist and the patient with halitosis. Chapter 11 in Bad Breath. Research Perspectives, Ramot Publishing Tel Aviv University, Isreal, 1995;pp. 175-188.

4. Rosenberg M, Kulkarni GV, Bosy A, et al. Reproducibility and sensitivity of oral malodor measurements with a portable sulphide monitor. $J$ Dent Res. 1991;70(11):1436-1140.

5. Geist H. Halitosis in ancient literature. Dent Abstr. 1957;2:417-418.
6. Grapp GL. Fetor oris (halitosis) A medical and dental responsibility. Northwest Med. 1993;32:375-380.

7. Prinz H. Offensive breath, its causes and its prevention. Dent Cosmos. 1930;72:700-707.

8. Vali A, Roohafza H, Keshteli AH, et al. Relationship between subjective halitosis and psychological factors. Int Dent J. 2015;65 (3):120-126.

9. Tonzetich J. Production and origin of oral malodor: a review of mechanisms and methods of analysis. J Periodontol. 1997;48(1):13-20.

10. Tonzetich J. Oral malodour: An indicator of health status and oral cleanliness. Int Dent. 1997;28(3):309-319.

11. Marsicano JA, de Moura-Grec PG, Bonato RC, et al. Gastroesophageal reflux, dental erosion, and halitosis in epidemiological surveys: a systematic review. Eur J Gastroenterol Hepatol. 2012;25(2):135-141.

12. Aung EE, Zaitsu T, Ueno M, Kawaguchi Y. Relationship of Oral Health Knoledge, Behavior and Status with Self-Perceived and Clinical Oral Malodor among Dental Patients. J Dent Healt Oral Disord Ther. 2015;3(2): 00083.

13. Wilhelm D, Himmelmann A, Axmann EM, et al. Clinical efficacy of a new tooth and tongue gel applied with a tongue cleaner in reducing oral halitosis. Quintessence Int. 2012;43(8):709-718. 